\title{
Haemodynamic Effects of Rapid Digitalization Following Acute Myocardial Infarction ${ }^{\star}$
}

\author{
RAPHAEL BALCON, JUDY HOY, AND EDGAR SOWTON \\ From the Institute of Cardiology and the National Heart Hospital, London W.1
}

Digitalis is now in common use following acute myocardial infarction, and the danger of producing arrhythmias appears to be no greater than in other groups of patients (Askey, 1951). The drug is frequently given intravenously for a rapid effect and to ensure that it will enter the circulation, but little is known of the immediate effects of such administration on the circulation. Malmcrona, Schröder, and Werkö (1966) have reported on 10 patients and we report here our results in a further 11 patients.

\section{SUBJECTS AND METHODS}

Eleven patients were studied from 12 hours to 5 days following an acute myocardial infarction, confirmed in all cases by electrocardiographic and serum enzyme changes. There were 7 men and 4 women, whose ages ranged from 39 to 67 , with a mean of 54.5 years. In all patients there was clinical evidence of poor left ventricular function. In 8 patients a third heart sound was present, in 2 there was an atrial sound, and in 4 the second heart sound was single or split paradoxically during respiration. Basal râles and crepitations were present in 3 subjects and the chest $x$-ray showed distended upper lobe veins in 2 . As a group, therefore, these patients showed early signs of left ventricular failure, but had not progressed to congestive cardiac failure. None of the patients had had previous digitalis therapy, and all were in sinus rhythm.

The study was carried out without disturbing the patients' normal routine. Pulmonary artery pressure was obtained via a fine flow-guided nylon catheter and aortic pressure via a similar teflon catheter, both introduced percutaneously. Cardiac output was measured by the dye-dilution technique, $5 \mathrm{mg}$. indocyanine green being injected into the pulmonary artery; arterial blood was sampled from the brachial artery and reinjected after each determination. Control readings were taken for at least 30 minutes before the intravenous injection of either $0.25 \mathrm{mg}$. acetyl beta strophanthidin ( 7 cases) or $0.5 \mathrm{mg}$. digoxin (4 cases). Further readings were taken 15, 30,

Received October 17, 1967.

* This work was supported in part by a grant from the British Heart Foundation. and 60 minutes after the injection. The parameters assessed were: mean pulmonary artery, mean aortic, and mean systolic ejection pressures, cardiac output, heart rate, and systolic ejection time. Stroke volume was derived by dividing cardiac output by heart rate; cardiac work by multiplying cardiac output by mean aortic pressure; stroke work by dividing cardiac work by heart rate; total peripheral resistance by dividing mean aortic pressure by cardiac output, and tension-time index as the product of mean systolic ejection pressure, systolic ejection time, and heart rate. The patients were lying flat throughout the study, with 2 pillows, and zero level for the pressure measurements was the mid-chest. Student's ' $t$ ' test was applied to the results to determine statistical significance.

\section{RESULTS}

The group means, and ranges for all the parameters measured, are shown in the Table.

There were no significant differences between the responses to acetyl strophanthidin and to digoxin, so the results have been considered together.

Heart Rate. The mean rate before digitalization was 82 beats/min. (range 70 to 90 ). Following digitalization the rate fell in 9 patients and increased slightly in the remaining 2 , the mean levels being 79 a minute after 30 minutes and 77 a minute after 60 minutes. The fall at 60 minutes was statistically significant $(0.02>p>0.01)$.

Cardiac Ouput. The mean cardiac output during control readings was $4.91 . / \mathrm{min}$. (range 3.5 to $6 \cdot 8$ ), and this was reduced in all 11 patients at some time during the 60 minutes after digitalization. The usual time sequence was an initial fall in output followed by a subsequent increase (Fig.), so that the mean level rose from $4 \cdot 2 \mathrm{l} / \mathrm{min}$. at 30 minutes $(0.02>\mathrm{p}>0.01)$ to $4.61 . / \mathrm{min}$. at 60 minutes $(0.1>p>0.05)$. 


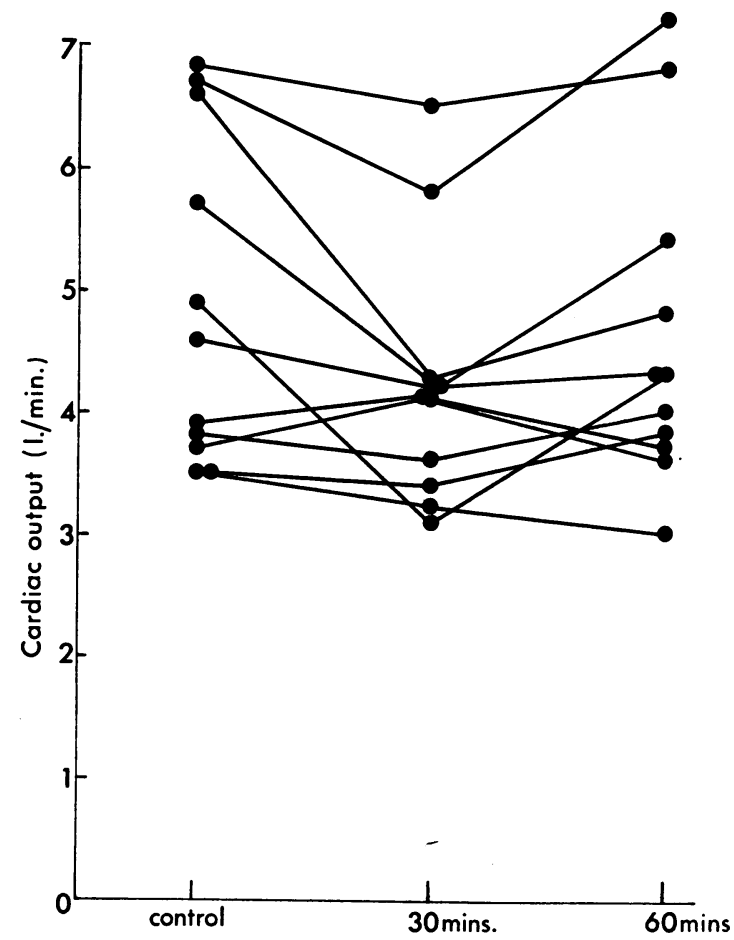

Fig.-Changes in cardiac output following acute digitalization. The output fell in all patients, usually with a subsequent increase.

Systemic Arterial Pressure. The aortic mean pressure changed very little following digitalization, the group mean for the control levels being 91 $\mathrm{mm} . \mathrm{Hg}$, with values of $80 \mathrm{~mm} . \mathrm{Hg}$ at 30 minutes and $93 \mathrm{~mm} . \mathrm{Hg}$ at 60 minutes. These changes are not statistically significant.

Cardiac Work. Since the cardiac output fell and the systemic arterial pressure remained approximately constant there was a fall in the calculated external work performed by the heart. This was statistically significant $(0.05>p>0.02) 30$ minutes after digitalization, the fall being 16 per cent at this time. By 60 minutes the minute work had returned to control levels.

Tension-time Index. This index decreased following digitalization, but the fall was less than 6 per cent at all times and was not statistically significant.

Other Parameters. Minor, non-significant changes occurred in the mean pulmonary artery pressure, systolic ejection time, and total peripheral resistance. The stroke volume fell by 7 per cent at 30 minutes, but this change did not reach statistical significance and the changes in stroke work were also just short of statistical significance $(0 \cdot 1>\mathrm{p}>0.05)$.

Side-effects. The electrocardiogram was monitored continuously during digitalization and throughout the subsequent period of study. No increased incidence of ectopic beats occurred and there were no dysrhythmias. In one patient the intravenous injection of $0.5 \mathrm{mg}$. digoxin was followed by ischaemic cardiac pain after 8 minutes, but no permanent electrocardiographic changes occurred, and there was no subsequent rise in serum enzymes. This episode of anginal pain was possibly provoked by the

TABLE

HAEMODYNAMIC EFFECTS OF RAPID DIGITALIZATION FOLLOWING ACUTE MYOCARDIAL INFARCTION

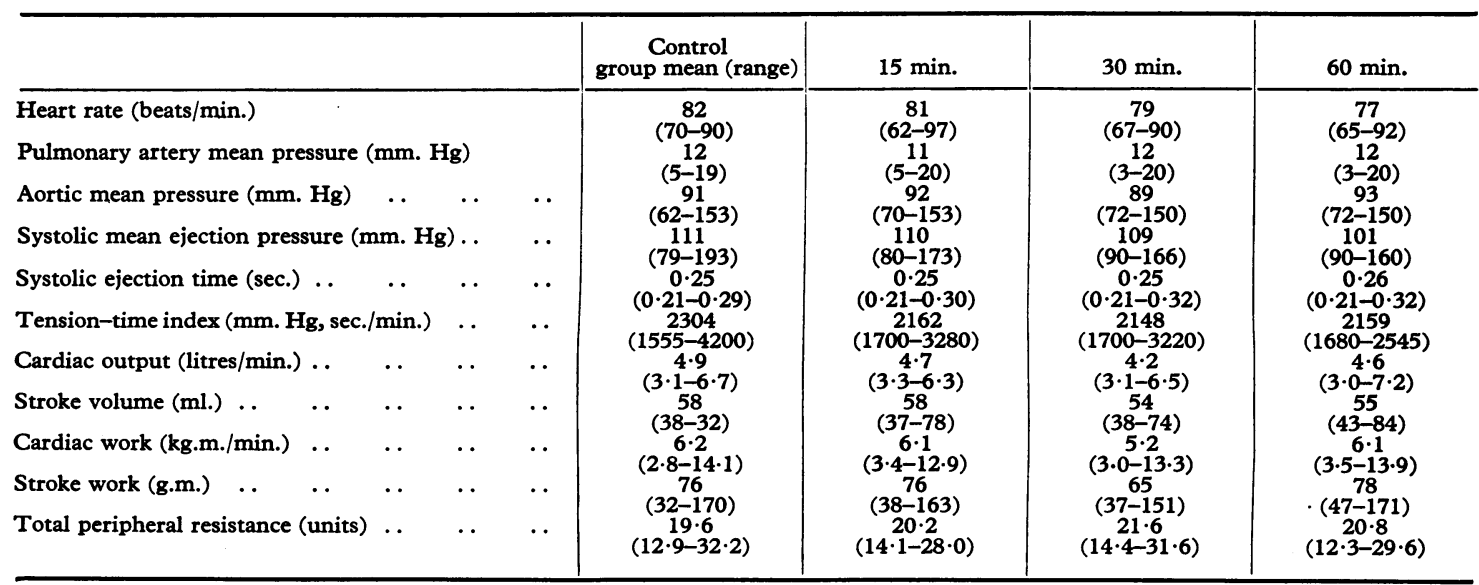


rapid digitalization, but proof of this relationship is not available, since this patient experienced spontaneous attacks of pain on other occasions, unrelated to drug therapy. No other side-effects were noted in any patient.

\section{Discussion}

Digitalis is known to increase cardiac contractile force and to result in an increased cardiac output in subjects with heart failure (Goodman and Gilman, 1965). Our patients all had evidence of poor left ventricular function, but were not in cardiogenic shock and did not have congestive cardiac failure. The mean cardiac output of our group before digitalization was not unduly low and the systemic blood pressure was well maintained. The 14 per cent fall in cardiac output which occurred following digitalis applies only to this group of patients, and it does not necessarily follow that a similar fall would occur in patients with shock. Digitalis reduces the cardiac output in normal hearts concomitantly with a reduction in ventricular volume (Goodman and Gilman, 1965), and it may be that this effect was occurring in our post-infarction patients. The minor reduction in heart rate we noted cannot entirely account for the fall in output, since the reduction in rate occurred after the maximum drop in output and continued at a time when output was rising again.

An increase in myocardial contractile force may be accompanied by an increased myocardial oxygen uptake, but the relationship between tension-time index and oxygen consumption by the heart is disturbed by inotropic interventions and by volume changes. We were unable to detect any evidence of a positive inotropic action with the simple methods available in this study. On the contrary there was a small fall in mean systolic ejection rate since the stroke volume was slightly reduced and the ejection time remained unchanged, but these changes were not statistically significant and so no firm conclusions can be drawn. Despite this failure to detect inotropic changes, it is not justifiable to interpret the constant tension-time index as indicating an unchanged myocardial oxygen uptake, since we have no data on cardiac volumes before and after digitalization.

The effects of rapid digitalization with acetyl strophanthidin in dogs with experimental cardiogenic shock produced by microsphere emboli of coronary arteries were studied by Cronin and Zsotér (1965). They reported a significant rise in arterial blood pressure and cardiac output with a reduction in left ventricular end-diastolic pressure, while control animals displayed only a rise in arterial pressure. The authors suggested that the haemodynamic effects of the drug were due to a peripheral vasoconstrictor action in addition to the positive inotropic action on the heart. Hood, McCarthy, and Lown (1967) studied dogs with experimental myocardial infarction produced by coronary artery ligation, and concluded that acetyl strophanthidin caused no improvement in left ventricular function. This conclusion is not in keeping with the report by Marano and co-workers (1965), who used the microsphere embolization method to produce acute myocardial infarction in dogs. These authors claimed that rapid digitalization with ouabain produced a beneficial but transitory improvement in cardiac output and aortic pressure in animals with shock, even in the absence of haemodynamic evidence of congestive cardiac failure. No such changes were found in control animals without infarction.

Malmcrona et al., studied 10 subjects with recent transmural myocardial infarction who were digitalized over 10 minutes with an intravenous injection of $0.8 \mathrm{mg}$. lanatoside $\mathrm{C}$, and found a rise of arterial pressure but no significant change in cardiac output or peripheral resistance. This group of patients differed from our own in that most of them had a substantial fall in brachial arterial pressure though they were stated not to be in left ventricular failure. The findings reported by Malmcrona, together with our own results, suggest that there is no haemodynamic benefit from digitalization of patients with acute myocardial infarction who are not shocked and are not in congestive cardiac failure. Our finding of a statistically significant fall in cardiac output 30 minutes after rapid digitalization may be related to the clinical state of our patients or possibly to the intravenous route of administration. It seems wise that if post-infarct patients are to be digitalized the drug should not be given intravenously, particularly since one of our patients may have experienced angina related to the injection.

\section{Summary AND CONCLUSIONS}

Eleven patients in sinus rhythm with recent myocardial infarction and showing evidence of poor left ventricular function were digitalized by the intravenous route. A statistically significant fall of cardiac output $(14 \%)$ occurred after 30 minutes with no change in pulmonary artery pressure, aortic pressure, or peripheral resistance. The output rose again subsequently but had not reached control levels by 60 minutes.

The heart rate fell following digitalization and the 6 per cent drop after 60 minutes was statistically significant. The fall in cardiac output was not solely due to the reduction in heart rate.

Since there was no evidence of a beneficial haemodynamic action of acute digitalization in this group, 
and angina may have been precipitated in one patient, we suggest that intravenous digitalization is contraindicated in patients of this type following myocardial infarction.

\section{REFERENCES}

Askey, J. M. (1951). Digitalis in acute myocardial infarction. F. Amer. med. Ass., 146, 1008.

Cronin, R. F.P., and Zsotér, T. (1965). Hemodynamic effects of rapid digitalization of experimental cardiogenic shock. Amer. Heart F., 69, 233.
Goodman, L. S., and Gilman, A. (1965). The Pharmacological Basis of Therapeutics, 2nd ed., pp. 673-681. Macmillan, New York.

Hood, W. B. Jr., McCarthy, B., and Lown, B. (1967). Myocardial infarction following coronary ligation in dogs: Hemodynamic effects of isoproterenol and acetyl strophanthidin. Circulat. Res., 21, 191.

Malmcrona, R., Schröder, G., and Werkö, L. (1966). Hemodynamic effects of digitalis in acute myocardial infarction. Acta med. scand., 180, 55.

Marano, A. J., Kline, H. J., Cestero, J., and Kuhn, L. A. (1965). The hemodynamic effects of ouabain in experimental acute myocardial infarction with shock. (Abstract.) Amer. F. Cardiol., 15, 139. 